\title{
Evolution and networks in ancient and widespread symbioses between Mucoromycotina and liverworts
}

\author{
William R. Rimington ${ }^{1,2,3}$ (1) - Silvia Pressel ${ }^{2}$ • Jeffrey G. Duckett ${ }^{2}$ • Katie J. Field ${ }^{4}$ - Martin I. Bidartondo ${ }^{1,3}$
}

Received: 29 May 2019 / Accepted: 13 September 2019/Published online: 13 November 2019

(C) The Author(s) 2019

\begin{abstract}
Like the majority of land plants, liverworts regularly form intimate symbioses with arbuscular mycorrhizal fungi (Glomeromycotina). Recent phylogenetic and physiological studies report that they also form intimate symbioses with Mucoromycotina fungi and that some of these, like those involving Glomeromycotina, represent nutritional mutualisms. To compare these symbioses, we carried out a global analysis of Mucoromycotina fungi in liverworts and other plants using species delimitation, ancestral reconstruction, and network analyses. We found that Mucoromycotina are more common and diverse symbionts of liverworts than previously thought, globally distributed, ancestral, and often co-occur with Glomeromycotina within plants. However, our results also suggest that the associations formed by Mucoromycotina fungi are fundamentally different because, unlike Glomeromycotina, they may have evolved multiple times and their symbiotic networks are un-nested (i.e., not forming nested subsets of species). We infer that the global Mucoromycotina symbiosis is evolutionarily and ecologically distinctive.
\end{abstract}

Keywords Fine root endophytes $\cdot$ Glomeromycotina $\cdot$ Liverworts $\cdot$ Mucoromycotina $\cdot$ Networks $\cdot$ Terrestrialization

\section{Introduction}

To what extent did fungi influence the conquest of land and greening of the planet by plants some 500 million years ago? This remains one of the most critical questions in land plant evolution ever since the idea of fungal-assisted plant terrestrialization was first proposed over 40 years ago

Electronic supplementary material The online version of this article (https://doi.org/10.1007/s00572-019-00918-x) contains supplementary material, which is available to authorized users.

William R. Rimington

w.rimington@gmail.com

Martin I. Bidartondo

m.bidartondo@imperial.ac.uk

1 Department of Life Sciences, Imperial College London, London SW7 2AZ, UK

2 Department of Life Sciences, Algae, Fungi and Plants Division, Natural History Museum, London, London SW7 5BD, UK

3 Comparative Plant and Fungal Biology, Royal Botanic Gardens, Kew, Richmond TW9 3DS, UK

4 Centre for Plant Sciences, Faculty of Biological Sciences, University of Leeds, Leeds LS2 9JT, UK
(Pirozynski and Malloch 1975). This hypothesis posits that Precambrian green algae, the ancestors of land plants, were able to colonize land successfully by entering into partnerships with fungi. The fungi provided early rootless plants with nutrients and water in exchange for photosynthesis-derived carbohydrates. Following terrestrialization, this relationship evolved into mycorrhizal symbioses, now present in more than $85 \%$ of plants (Brundrett and Tedersoo 2018). Of these, arbuscular mycorrhizas (AM) formed by Glomeromycotina fungi are by far the most widespread, occurring in at least $72 \%$ of vascular plants (Brundrett and Tedersoo 2018).

Liverworts are one of the three groups of bryophytes, or non-vascular plants, alongside mosses and hornworts. Though the order of divergence of these groups remains under active debate (Puttick et al. 2018; Rensing 2018; de Sousa et al. 2019), bryophytes are generally regarded as some of the earliest terrestrial plants (Renzaglia et al. 2018) and have provided invaluable insights into the origin and evolution of key land plant innovations including mycorrhizas (e.g., Wang et al. 2010; Field et al. 2015a, b, 2016, 2019). Indeed, Glomeromycotina colonization in liverworts (Ligrone et al. 2007), together with the dominance of AM in extant land plant lineages, and their putative occurrence in fossils (Taylor et al. 1995) have long supported the paradigm that 
Glomeromycotina formed the ancestral plant-fungus symbiosis (Smith and Read 2008). However, this consensus, dating over 20 years, was challenged in 2011 by a proposal, using liverworts as a proxy for the first land plants, that Glomeromycotina-plant mutualism was predated by symbioses involving Endogonales belonging to Mucoromycotina (Bidartondo et al. 2011). Supporting physiological and phylogenetic evidence has since accumulated rapidly (Field et al. 2015a; Feijen et al. 2018; Hoysted et al. 2018). Mucoromycotina fungi have been shown to colonize the earliest-diverging liverwort clade, the Haplomitriopsida (Bidartondo et al. 2011), Koch's postulates have been fulfilled, and isotope tracer experiments have demonstrated bidirectional nutritional exchange between some Haplomitriopsida and Mucoromycotina (Field et al. 2015b). Mucoromycotina fungi also colonize hornworts (Desirò et al. 2013) and the earliest-diverging vascular plants, lycophytes, and ferns (Rimington et al. 2015), indicating that these fungi are widespread symbionts of ancient plant lineages. Hornworts, lycophytes, and some non-Haplomitriopsida liverworts (Marchantiopsida and Pelliidae) are simultaneously colonized by both Mucoromycotina and Glomeromycotina; in liverworts, these "dual" colonizations have been shown to be more nutritionally beneficial than those involving only one fungal lineage (Field et al. 2016, 2019).

While the Glomeromycotina have been studied intensively for decades, the Mucoromycotina remain relatively poorly understood. Mucoromycotina and Glomeromycotina have recently been placed in the phylum Mucoromycota (Spatafora et al. 2016, but see Tedersoo et al. 2018), having been previously either an unplaced subphylum (Mucoromycotina) or a monophyletic phylum (Glomeromycota) (Schüßler et al. 2001; Hibbett et al. 2007). Of the three Mucoromycotina orders (Endogonales, Mucorales, and Umbelopsidales), only members of Endogonales (Endogonaceae and Densosporaceae) are known to enter into symbioses with plants (Desirò et al. 2017), being common endosymbionts of early-diverging lineages (liverworts, hornworts, lycophytes, and ferns) (Bidartondo et al. 2011; Desirò et al. 2013; Rimington et al. 2015) with some members ectomycorrhizal with trees (Walker 1985; Desirò et al. 2017; Yamamoto et al. 2017). Recent reports indicate that fine root endophytes, arbuscule-forming fungi found throughout vascular plants (Orchard et al. 2017a), may also be members of the Mucoromycotina rather than Glomeromycotina (Orchard et al. 2017b; Walker et al. 2018). Therefore, our understanding of the host range of Mucoromycotina across land plants and appreciation of their potential significance in modern ecosystems is expanding rapidly.

Here, we present a worldwide analysis of symbiotic Mucoromycotina associating with liverworts-the Haplomitriopsida, Marchantiopsida (complex thalloid), and Pelliidae (simple thalloid). Through DNA sequencing of $18 \mathrm{~S}$
rDNA, species delimitation, and ancestral reconstruction, we aimed to shed light on this symbiosis by revealing its diversity and global distribution and comparing it to the symbiosis formed by Glomeromycotina (Rimington et al. 2018). We also present a first network analysis of fungal interactions with non-vascular plants. Network analysis allows visualization and quantification of how members of a network interact and it has become popular across biotic interactions, including mycorrhizas, to show which plants interact with which fungi, and vice versa, and to infer symbiotic ecology and evolution (Southworth et al. 2005; Bascompte and Jordano 2014; van der Heijden et al. 2015). Plant and fungal taxa represent nodes, and patterns of fungal occurrences are links connecting the two sets (fungi vs. plants or vice versa) of nodes. Once nodes and links are established, mycorrhizal network architecture can be quantified and compared.

\section{Methods}

\section{Plant material and symbiotic fungi}

We focused on liverwort clades (Haplomitriopsida, Marchantiopsida (complex thalloids), and Pelliidae (simple thalloids, within Jungermanniopsida)) with Mucoromycotina associations. The largest group of liverworts, the leafy Jungermanniidae, forms associations only with ascomycetes or basidiomycetes or lack fungi (Pressel et al. 2010). Liverwort collection sites were in 24 countries and all continents except Antarctica (Table S1). In total, 674 mature liverwort gametophytes were newly collected from the classes Haplomitriopsida (72 samples), Marchantiopsida (411), and Pelliidae (191). Specimen vouchers have been deposited at the Natural History Museum, London. Using the latest nomenclatures, samples were assigned to 85 species (Soderstrom et al. 2016; Stotler and Crandall-Stotler 2017). Only genus-level identification was possible for 49 samples, so the total number of species is likely higher than 85 . Within 3 days of collection liverwort samples were cleaned of soil and debris using water and forceps. Then, the thallus midrib was dissected as this is where fungal colonization is highest (Pressel et al. 2010). Dissection was performed by removing the wings and rhizoids and cutting thallus sections ca. $3 \mathrm{~mm}^{2}$ that were placed in CTAB buffer and stored at $-20^{\circ} \mathrm{C}$.

Liverwort sections were used for sequencing the $18 \mathrm{~S}$ ribosomal RNA gene of Mucoromycotina fungi. This gene was selected as it is the norm for investigating Mucoromycotina in plants (Bidartondo et al. 2011; Desirò et al. 2013; Rimington et al. 2015) and allows comparison with previous studies. The advantages and disadvantages of molecular detection using the $18 \mathrm{~S}$ for endomycorrhizas have been reviewed by Öpik et al. (2014). Genomic DNA extraction was performed using chloroform extraction (Gardes and Bruns 1993) and the 
GeneClean II kit (QBioGene). After extraction, fungal DNA was amplified using the universal fungal primers NS1 (White et al. 1990) and EF3 (Smit et al. 1999) and JumpStart (Sigma) using the following PCR settings: $94^{\circ} \mathrm{C}$ for $2 \mathrm{~min} ; 34$ cycles of $94{ }^{\circ} \mathrm{C}$ for $30 \mathrm{~s}, 53{ }^{\circ} \mathrm{C}$ for $30 \mathrm{~s}$, and $72{ }^{\circ} \mathrm{C}$ for $1 \mathrm{~min} 30 \mathrm{~s}$; $72^{\circ} \mathrm{C}$ for $7 \mathrm{~min}$. Following PCR, products were cloned with the Invitrogen TOPO TA Cloning Kit. We re-amplified DNA from between four and eight $E$. coli colonies per sample using JumpStart and NS1/EF3 with the following PCR settings: $94{ }^{\circ} \mathrm{C}$ for $7 \mathrm{~min} ; 25$ cycles of $94{ }^{\circ} \mathrm{C}$ for $30 \mathrm{~s}, 53{ }^{\circ} \mathrm{C}$ for $30 \mathrm{~s}$, and $72{ }^{\circ} \mathrm{C}$ for $1 \mathrm{~min} 30 \mathrm{~s} ; 72{ }^{\circ} \mathrm{C}$ for $5 \mathrm{~min}$. The products of this second PCR were prepared for sequencing using ExoSAP-IT (Affymetrix) and BigDye v. 3.1 (Applied Biosystems) with the NS1 primer. For sequencing, an ABI3730 genetic analyzer was used (Applied Biosystems). The NCBI BLAST was used to assign DNA sequences (ca. $600 \mathrm{bp}$ ) to subphyla, and those found to be Mucoromycotina were further sequenced using the primers NS3 and NS5 (White et al. 1990). Sequences (ca. $1600 \mathrm{bp}$ ) were edited and assembled into contigs using Geneious v. 7 (Kearse et al. 2012). In cases where more than one clone from a sample was identified as Mucoromycotina, the sequences were aligned, and if they shared a pairwise similarity of more than $98 \%$, then only one was selected for full sequencing of the $18 \mathrm{~S}$ gene. The MUSCLE alignment algorithm (Edgar 2004) was used in MEGA (Kumar et al. 2016). The programs UCHIME (Edgar et al. 2011) and UNOISE2 (Edgar 2016) were used to test for chimeras.

\section{Phylogenetic analysis and species delimitation}

The sequences produced in this investigation were combined with Mucoromycotina sequences from previous investigations of liverworts (Bidartondo et al. 2011; Field et al. 2015b, 2016), hornworts (Desirò et al. 2013), lycophytes, and ferns (Rimington et al. 2015), as well as sequences from Endogonales fruitbodies. Throughout these analyses, after the sequences were aligned, MEGA was used to test evolutionary models and for producing maximum likelihood phylogenies. Bayesian inference was performed using MrBayes (Huelsenbeck and Ronquist 2001) and outputs visualized using FigTree v. 1.4 (Rambaut 2012).

Two species delimitation methods, Poisson Tree Processes (PTP) and Generalized Mixed Yule Coalescent (GMYC), were used to group Mucoromycotina sequences into taxa. These methods are both superior to sequence-similarity OTU-calling methods as they incorporate phylogenetic analyses. Prior to these analyses, ALTER was used to remove haplotype sequences (Glez-Pena et al. 2010); haplotypes create zero branch lengths which can negatively influence analyses (Fujisawa and Barraclough 2013). The PTP is the simpler of the two methods and uses a rooted phylogenetic tree to model speciation rate (Zhang et al. 2013). This model utilizes the branch lengths of the tree with the assumption that each substitution creates a probability of speciation, as such, as the number of substitutions increases so does the probability of speciation. The input trees for this analysis were created using RAxML (Stamatakis 2014) which was utilized using RAxML-HPC2 on XSEDE on the CIPRES scientific gateway with 1000 bootstrap iterations (Miller et al. 2010). The PTP was performed using an updated version of the original analysis called mptp (Kapli et al. 2017) which is able to use Markov Chain Monte Carlo (MCMC) sampling (in all cases $10,000,000$ generations were run) to produce support values for the groupings produced during delimitation. The GMYC method is more complex than PTP; it requires an unrooted, ultrametric, time-calibrated phylogenetic tree as input. The model then uses branch lengths to differentiate between within-population coalescence and speciation events (Fujisawa and Barraclough 2013). Input trees were produced using BEAST2 (Bouckaert et al. 2014) run through CIPRES. Priors were added to the BEAST analysis using BEAUTi (Drummond and Rambaut 2007). In all analyses, the molecular clock was "Relaxed Clock Log Normal" and the population model was "Birth Death." The bModelTest was utilized to select the evolutionary model (Bouckaert and Drummond 2017). The number of generations in the MCMC chain was influenced by the size of the alignment and the convergence success and ranged between 10,000,000 and 200,000,000. Tree sampling frequency was determinant upon MCMC chain length to ensure a total of 1000 trees were sampled during the analysis. Tracer v1.6 was used to visualize the BEAST outputs (Rambaut et al. 2014). Convergence was deemed to be successful if all effective sample size values were greater than 200. If any were below this value, the BEAST analysis was run again using an increased chain length. TreeAnnotator was run on CIPRES to convert the 1000 trees into one consensus tree using a 10\% burn-in, common ancestor heights, and maximum clade credibility as the analysis settings (Drummond and Rambaut 2007). This consensus tree was imported into RStudio (RStudio Team 2015) using the "rncl" package, after which GMYC was run using the "splits" package. Like mptp, "splits" has the capability to calculate the confidence in the delimited groups produced. Singletons are known to influence these analyses so in cases where singletons were delimited by GMYC, the full analysis was run again with singleton sequences removed from the alignment. The results of these delimitation methods were used to assign sequences to "early-diverging plant-Mucoromycotina taxa" (epMT) (i.e., fungi associated with early-diverging plants). When mptp and GMYC did not agree on the groupings, the group with the highest confidence level was selected. Species accumulation curves were produced using the "vegan" package within RStudio. Two curves were produced: one containing only epMT and the other containing both epMT and singletons. Extrapolation of the number of taxa was performed using the bootstrap method. 
The epMT were combined with the epGT (early-diverging plant Glomeromycotina taxa) previously detected in these plants (Rimington et al. 2018) to allow comparison of Mucoromycotina and Glomeromycotina. The method of producing epGT was the same as used when assigning epMT so these results are directly comparable. The epGT were used in network analysis and ancestral reconstruction of fungal symbioses in liverworts.

\section{Ancestral reconstruction}

The ancestral state of fungal symbioses in liverworts was reconstructed using Mesquite v. 3.31 (Maddison and Maddison 2017) with a representative of each liverwort genus sampled. Prior to ancestral reconstruction, a liverwort phylogenetic tree was produced using $26 \mathrm{~S}$ and trnK-psbA spacer sequences from GenBank (Fig. S3). Where possible, these sequences represented the most frequently analyzed species of the genus in this investigation. For some genera, only genes from species not included in this investigation were available; however, as the purpose of the tree was to represent the phylogenetic placement of genera relative to each other, this was not considered important. There are no published sequences of the Pelliidae liverwort Sewardiella so sequences from its closest related genus, Petalophyllum, were used in the analyses. As these two genera are the only two members of the family Petalophyllaceae, this should not influence the topology. The moss Takakia ceratophylla was included as an outgroup. Following alignment in MEGA, Bayesian inference was performed using MrBayes with the nst $=6$ model, invgamma rates, and 10,000,000 MCMC generations. The phylogenetic tree was imported into Mesquite and the ancestral reconstruction was performed separately for Mucoromycotina and Glomeromycotina. The liverwort genera were scored for presence or absence of fungi based on the DNA sequencing results of this study (Mucoromycotina) and the results of Rimington et al. (2018) (Glomeromycotina). Ancestral reconstruction was performed using maximum likelihood and maximum parsimony. The Asymmetry Likelihood Ratio Test in Mesquite determined the best model to use was the Markov 1-parameter model. The results of the analyses for both Mucoromycotina and Glomeromycotina were viewed simultaneously using the mirror tree window in Mesquite. A second liverwort phylogeny was produced for these analyses based on phylogenies that used five (Forrest et al. 2006) and eleven genetic markers (Flores et al. 2017). This was done because the original tree produced using $26 \mathrm{~S}$ and the trnK-psbA spacer contained small topological differences from trees that used a larger number of genes. Starting with the original, "uncorrected" phylogeny, Mesquite's branch moving tool was used to reorder branches based on the larger phylogenies while maintaining tree branch lengths. Ancestral reconstruction analyses were run again on this new tree using the same settings as before.

\section{Network analysis}

Network analysis was performed on liverwort samples collected from the South Island of New Zealand, the most sampled location and with the largest number of country-specific epMT (taxa only found in one country and none of the other countries sampled in this study). The presence of epMT endemic to islands means that network analysis on a larger scale would not be valid. Network analysis consisted of four parts: (1) visualization, (2) connectance, (3) nestedness, and (4) modularity. For each of these, three separate analyses were performed, a network consisting of liverworts and both fungal lineages (combined-network) and two networks consisting of liverworts and only one fungal lineage (Glomeromycotinaonly and Mucoromycotina-only). (1) The network was visualized in RStudio using "igraph" and the FruchtermanReingold layout. The degree function was used to relate node size to connection number while the weight function was used to relate connector thickness to the number of times the association was observed. (2) Connectance is the proportion of observed interactions out of the number that are theoretically possible and was calculated in RStudio using the "bipartite" package. (3) Nestedness measures the extent to which specialists in a network interact with generalists. A number of methods have been proposed to measure this character including Matrix Temperature (T), Nestedness measure based on Overlap and Decreasing Fills (NODF), and the Brualdi and Sanderson metric (BR). The most widely used nestedness metric is $\mathrm{T}$ which measures the extent to which the matrix departs from perfect nestedness, a value of $0^{\circ}$ denotes a perfectly nested network and $100^{\circ}$ is perfectly un-nested (Atmar and Patterson 1993). The NODF method calculates values of nestedness for rows and columns in a matrix by producing an average nestedness value from all combinations of pairs of rows and columns. These values are then combined to provide a value for the whole matrix (Almeida-Neto et al. 2008). The BR metric is a count of the smallest number of absences or presences which must be removed to create a perfectly nested matrix (Brualdi and Sanderson 1999). The NODF and BR are considered to be superior to $T$ (Strona et al. 2014). Greater details of these concepts are reviewed in Ulrich et al. (2009). The programs NeD (T, NODF, and BR) and ANIHADO (T and NODF) were used to calculate these nestedness values (Guimarães and Guimarães 2006; Strona et al. 2014). For these calculations presence/absence matrices were used that were first ordered based on the sums of marginal rows and columns with the most common in the top left of the matrix, as is necessary in nestedness calculations (Ulrich et al. 2009). To assess the significance of the nestedness scores, null model replicates were run to allow comparisons. The CE null model was used (null model 2) in both NeD and ANIHADO with 999 random network replicates. In this model, the probability of a matrix cell being occupied is proportional to the row and 
column total (Bascompte et al. 2003). (4) Modularity detects the presence of modules in a network and the extent to which the presence of modules is a character of the network. Modules are groups of nodes that are more linked to each other than they are to other nodes or modules. In modularity analysis, nodes are grouped to maximize the number of links within modules and minimize the number of links between modules. NetCarto was used to detect modules by simulated annealing to maximize modularity and assign roles to nodes (Guimera and Amaral 2005) with an initial temperature of 10, an iteration factor of 1.0 , and a 0.999 cooling factor. Modularity significance was tested within NetCarto using 100 randomizations (Guimera et al. 2004). The average modularity value and standard deviation of these randomizations were used to produce a one-way $Z$ value for significance of modularity. NetCarto can also assign roles to nodes within modules by producing a value for participation coefficient (PC) and within-module relative degree (RD) for each node. The assigned roles (ultra-peripheral node, peripheral node, non-hub connector, or connector hub) were labeled specialists if $\mathrm{PC} \leq 2.5$ and $\mathrm{RD} \leq 0.62$ (Olesen et al. 2007). If $\mathrm{PC}>2.5$ and/or $\mathrm{RD}>0.62$, then the node was labeled a generalist.

\section{Results}

\section{Mucoromycotina colonization is widespread in liverworts}

Mucoromycotina were detected in $24 \%$ of the 674 liverwort samples and were found in sixteen countries and all six continents investigated. At the level of class/subclass colonization rates were Haplomitriopsida 69\%, Marchantiopsida 14\%, and Pelliidae 31\%. Both Haplomitriopsida genera, Treubia and Haplomitrium (six species), six Marchantiopsida genera (13 species), and eight Pelliidae genera (17 species) were colonized by Mucoromycotina. Full results including fungal taxa detected and collection details are in Table S1. Results and detection rates for each liverwort group summarized at the genus level are in Table 1. Comparing our results with those of a worldwide analysis of Glomeromycotina in these plants (Rimington et al. 2018) indicates that many liverwort species can be colonized by both fungal lineages (Table 1), often simultaneously within the same plant individual (co-colonized). In total, 42 liverwort samples (15 Marchantiopsida, 27 Pelliidae), representing 21 species from 12 genera (six Marchantiopsida and six Pelliidae), were co-colonized by both Mucoromycotina and Glomeromycotina. Every Marchantiopsida genus harboring Mucoromycotina symbionts can be co-colonized by both fungal lineages. The Haplomitriopsida are the only group to form associations exclusively with Mucoromycotina. The most frequently cocolonized genus was Fossombronia, 22 samples belonging to eight species. The Mucoromycotina and Glomeromycotina that co-colonize liverworts are diverse and co-colonization is not limited to specific fungal taxa.

\section{Diverse Mucoromycotina taxa colonize early-diverging land plants}

Species delimitation using Generalized Mixed Yule Coalescent (GMYC) and multi-rate Poisson Tree Process (mPTP) placed the Mucoromycotina detected in liverworts, hornworts, lycophytes, and ferns into 36 taxa (epMT, earlydiverging plant-Mucoromycotina taxa), summarized in Fig. 1 (see Fig. S1 for support values for this tree). Species accumulation curves indicate that $83-88 \%$ of the endosymbiotic Mucoromycotina that colonize the liverworts analyzed have been detected (Fig. S2). Eight of the 36 epMT are exclusive to liverworts, one to lycophytes, one to hornworts, and three to lycophytes and hornworts. Liverworts were colonized by more epMT (31 epMT) than hornworts (24 epMT), lycophytes ( 5 epMT), and ferns ( 2 epMT). Fifteen sequences were delimited as singletons; ten from liverworts, four from hornworts, and one from a lycophyte.

The majority of epMT have been assigned to the two Endogonales families (Endogonaceae and Densosporaceae). We detected at least six potentially new Endogonales genera, i.e., strongly supported clades in phylogenetic trees that do not contain representatives of any described Endogonales. There is little overlap between sequences of fruiting bodies and plant-colonizing Mucoromycotina. Only five of the epMT contained sequences from Mucoromycotina fruitbodies (epMT23, 27, 28, 29, and 36); the endosymbiotic Mucoromycotina that are members of these five epMT predominately originated from Haplomitriopsida liverworts, in particular Haplomitrium, or hornworts.

\section{Mucoromycotina formed the ancestral liverwort-fungal symbiosis}

Ancestral reconstruction using maximum likelihood analysis supports Mucoromycotina symbiosis as an ancestral state for all liverworts and that both Mucoromycotina and Glomeromycotina are ancestral symbionts for the nonHaplomitriopsida liverworts (Fig. 2). Maximum parsimony analysis also supports these ancestral states. There has been only one gain of Glomeromycotina during the evolution of liverworts, which occurred in the ancestor of all the nonHaplomitriopsida liverworts. This gain has been followed by five loss events, impacting six of the genera analyzed (Phyllothallia, Oxymitra, Cryptomitrum, Corsinia, Cyathodium, and Sauteria), but there have been no additional gains. On the other hand, Mucoromycotina symbiosis in liverworts has likely undergone a number of losses and reacquisitions. As well as the well-documented loss of fungal 
Table 1 Mucoromycotina detection in liverworts

\begin{tabular}{|c|c|c|c|c|c|}
\hline & & Sample no. & No. colonized by Mucoromycotina & Species no. & Colonized by Glomeromycotina? \\
\hline All samples & & 674 & $165(24 \%)$ & $\geq 85$ & \\
\hline Haplomitriopsida & & 72 & $50(69 \%)$ & 7 & \\
\hline \multirow[t]{2}{*}{ Treubiales } & Treubiaceae & & & & \\
\hline & Treubia & 56 & $42(75 \%)$ & 2 & No \\
\hline \multirow[t]{2}{*}{ Calobryales } & Haplomitriaceae & & & & \\
\hline & Haplomitrium & 16 & $8(50 \%)$ & 5 & No \\
\hline Marchantiopsida & & 411 & $56(14 \%)$ & 46 & \\
\hline \multirow[t]{2}{*}{ Neohodgsoniales } & Neohodgsoniaceae & & & & \\
\hline & Neohodgsonia & 8 & $3(38 \%)$ & 1 & Yes* \\
\hline \multirow[t]{2}{*}{ Lunulariales } & Lunulariaceae & & & & \\
\hline & Lunularia & 36 & $9(25 \%)$ & 1 & Yes* \\
\hline \multirow[t]{27}{*}{ Marchantiales } & Marchantiaceae & & & & \\
\hline & Marchantia & 63 & 0 & $\geq 10$ & Yes \\
\hline & Preissia & 9 & 0 & 1 & Yes \\
\hline & Aytoniaceae & & & & \\
\hline & Asterella & 81 & $24(30 \%)$ & $\geq 12$ & Yes* \\
\hline & Cryptomitrium & 6 & 0 & 2 & No \\
\hline & Mannia & 5 & 0 & $\geq 1$ & Yes \\
\hline & Plagiochasma & 48 & $6(13 \%)$ & $\geq 2$ & Yes* \\
\hline & Reboulia & 9 & 0 & 1 & Yes \\
\hline & Cleveaceae & & & & \\
\hline & Athalamia & 1 & 0 & 1 & Yes \\
\hline & Clevea & 2 & 0 & 1 & Yes \\
\hline & Sauteria & 2 & 0 & 1 & No \\
\hline & Conocephalaceae & & & & \\
\hline & Conocephalum & 30 & 0 & 3 & Yes \\
\hline & Cyathodiaceae & & & & \\
\hline & Cyathodium & 7 & 0 & $\geq 3$ & No \\
\hline & Corsiniaceae & & & & \\
\hline & Corsinia & 4 & 0 & 1 & No \\
\hline & Oxymitraceae & & & & \\
\hline & Oxymitra & 1 & 0 & 1 & No \\
\hline & Targioniaceae & & & & \\
\hline & Targionia & 34 & $10(29 \%)$ & 1 & Yes* \\
\hline & Monocleaceae & & & & \\
\hline & Monoclea & 34 & $4(12 \%)$ & 2 & Yes* \\
\hline & Dumortieraceae & & & & \\
\hline & Dumortiera & 31 & 0 & 1 & Yes \\
\hline \multicolumn{2}{|l|}{ Pelliidae } & 191 & $59(31 \%)$ & $\geq 32$ & \\
\hline \multirow[t]{4}{*}{ Pelliales } & Noterocladaceae & & & & \\
\hline & Noteroclada & 4 & 0 & 1 & Yes \\
\hline & Pelliaceae & & & & \\
\hline & Pellia & 16 & $1(6 \%)$ & 3 & Yes \\
\hline \multirow[t]{5}{*}{ Fossombroniales } & Calyculariaceae & & & & \\
\hline & Calycularia & 10 & $3(30 \%)$ & 1 & Yes* \\
\hline & Sewardiella & 3 & $2(67 \%)$ & 1 & Yes* \\
\hline & Allisoniaceae & & & & \\
\hline & Allisonia & 7 & $1(14 \%)$ & 1 & Yes* \\
\hline
\end{tabular}


Table 1 (continued)

\begin{tabular}{|c|c|c|c|c|c|}
\hline & & Sample no. & No. colonized by Mucoromycotina & Species no. & Colonized by Glomeromycotina? \\
\hline \multirow{12}{*}{ Pallaviciniales } & Fossombronia & 116 & $49(42 \%)$ & $\geq 15$ & Yes* \\
\hline & Phyllothalliaceae & & & & \\
\hline & Phyllothallia & 3 & 0 & 1 & No \\
\hline & Moerckiaceae & & & & \\
\hline & Moerckia & 1 & $1(100 \%)$ & 1 & Yes* \\
\hline & Hymenophytaceae & & & & \\
\hline & Hymenophyton & 4 & 0 & 1 & Yes \\
\hline & Pallaviciniaceae & & & & \\
\hline & Jensenia & 1 & 0 & 1 & Yes \\
\hline & Pallavicinia & 5 & $1(20 \%)$ & 1 & Yes* \\
\hline & Podomitrium & 2 & 0 & 1 & Yes \\
\hline & Symphyogyna & 19 & $1(5 \%)$ & 4 & Yes \\
\hline
\end{tabular}

The number in brackets is the Mucoromycotina detection rate for the group/genus. The greater than or equal to symbol indicates some samples could only be identified to genus level, so it is the minimum number of species. Glomeromycotina colonization is based on Rimington et al. (2018). An asterisk indicates that individuals within the genus were found to be co-colonized by both fungal lineages

symbioses in Blasiales and Sphaerocarpales (Pressel et al. 2010), there appears to have been a major loss from the Marchantiales with a subsequent regain in four of the genera analyzed (Targionia, Asterella, Plagiochasma, and Monoclea). Mucoromycotina symbiosis appears to be the ancestral state for Pelliidae with losses in Noteroclada, Phyllothalia, Hymenophyton, Jensenia, and Podomitrium. Analysis of the "uncorrected" liverwort tree (Fig. S3), despite its slightly altered topology, supports identical ancestral states and losses and gains of both Glomeromycotina and Mucoromycotina. Presence/absence of fungal families in liverwort genera (Fig. 2) reveals that Fossombronia engages in the most diverse interactions with both fungal lineages. Treubia was only found to associate with two Mucoromycotina families, despite having the highest Mucoromycotina detection rate.

\section{Contrasting Mucoromycotina and Glomeromycotina networks}

The network shared between New Zealand (South Island) liverworts and Glomeromycotina and Mucoromycotina (combinednetwork) is shown in Fig. 3. Alternative visualizations, including Glomeromycotina- and Mucoromycotina-only networks, are shown in Fig. S4. The combined-network had a connectance of $8 \%$ while the Glomeromycotina- and Mucoromycotina-only networks each had a connectance of $11 \%$ and $13 \%$, respectively. The different nestedness calculation methods did not always agree on whether the networks were nested (Table 2). The only consensus from all three methods was that the Mucoromycotinaonly network is not nested. For the combined-network, NODF and $\mathrm{T}$ support the network is significantly nested. The Glomeromycotina-only network was supported as significantly nested by T and BR. Furthermore, when looking at the plants and fungi individually, the NODF method significantly supports that both the host and symbiont components of the network are nested in the combined- and Glomeromycotina-only networks. These results suggest that the combined- and Glomeromycotinaonly networks are nested but the Mucoromycotina-only network is not. None of the three networks were found to be significantly modular (Table 2). The modularity analysis determined that the networks are dominated by specialists and not generalists, with specialists representing $86-94 \%$ of the nodes in the three networks (Table S2a). Fossombronia pusilla was the only connector hub in all three networks. Monoclea forsteri and Lunularia cruciata were connector hubs in the combined-network, likely due to their dual colonizations. The other generalist nodes identified (non-hub connectors) were both plants and fungi and depended upon the network in question. Detailed network analysis values are in Table S2a.

\section{Discussion}

Mucoromycotina symbionts have been found throughout the early-diverging liverworts. Analysis of the networks shared between symbiotic fungi and their liverwort hosts suggests that Mucoromycotina symbiosis shares more similarities with ectomycorrhizas (ECM) than arbuscular mycorrhizas (AM).

\section{Diverse Mucoromycotina fungi colonize early-diverging land plants}

Prior to this study nine liverwort species had been confirmed molecularly to host Mucoromycotina (Bidartondo 


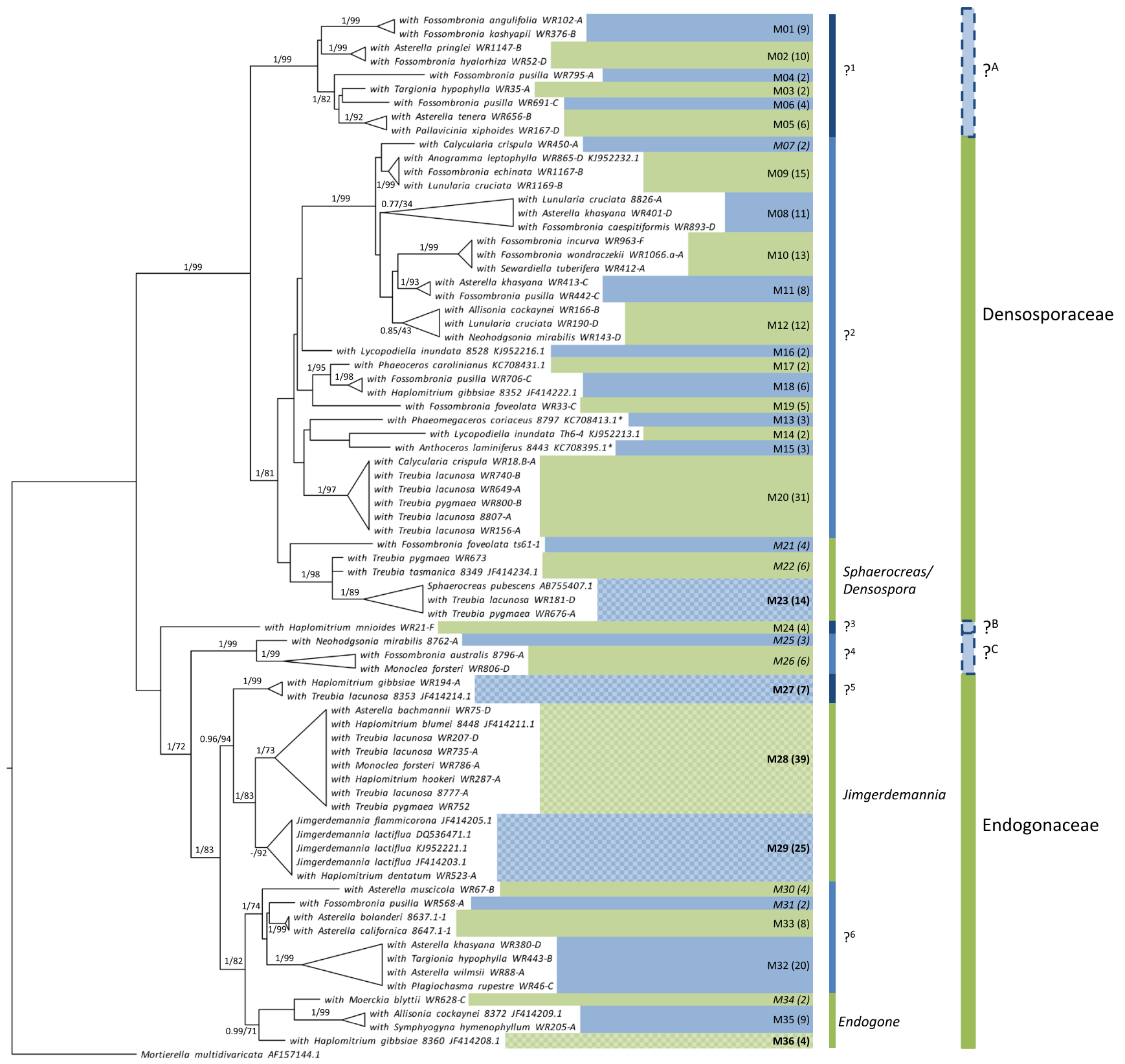

Fig. 1 Diverse Mucoromycotina taxa colonize early-diverging plants. Maximum likelihood phylogeny of the Mucoromycotina that colonize liverworts and the results of species delimitation (epMT labels have been shortened to M) based on 18S DNA sequences. Support values are the result of both Bayesian inference and 1000 bootstrap replicates. Only support values for the main branches are provided-full support values and analysis settings are detailed in Fig. S1. A dash indicates Bayesian

et al. 2011). Our study has increased this number to 39 . Furthermore, we have more than doubled the total number of plant species confirmed to enter into endosymbioses with Mucoromycotina: 39 liverworts, 15 hornworts (Desirò et al. 2013), four lycophytes, one fern (Rimington et al. 2015), and one angiosperm (Orchard inference did not agree with maximum likelihood. Figures in brackets indicate the number of DNA sequences that belong to each epMT. The epMT in bold include sequences from Endogonales fruitbodies. Italicized epMT are specific to liverworts. Genus and family labels are based on Desirò et al. (2017). Question marks indicate putative new fungal genera (1-6) and families (A-C). Alternating blue and green are used to highlight different clades

et al. 2017b) though diverse vascular plants have been reported to harbor fine root endophytes (Orchard et al. 2017a). The Haplomitriopsida were the only plants exclusively colonized by Mucoromycotina, in line with previous reports (Bidartondo et al. 2011; Field et al. 2015b). All other liverwort genera found to associate with 


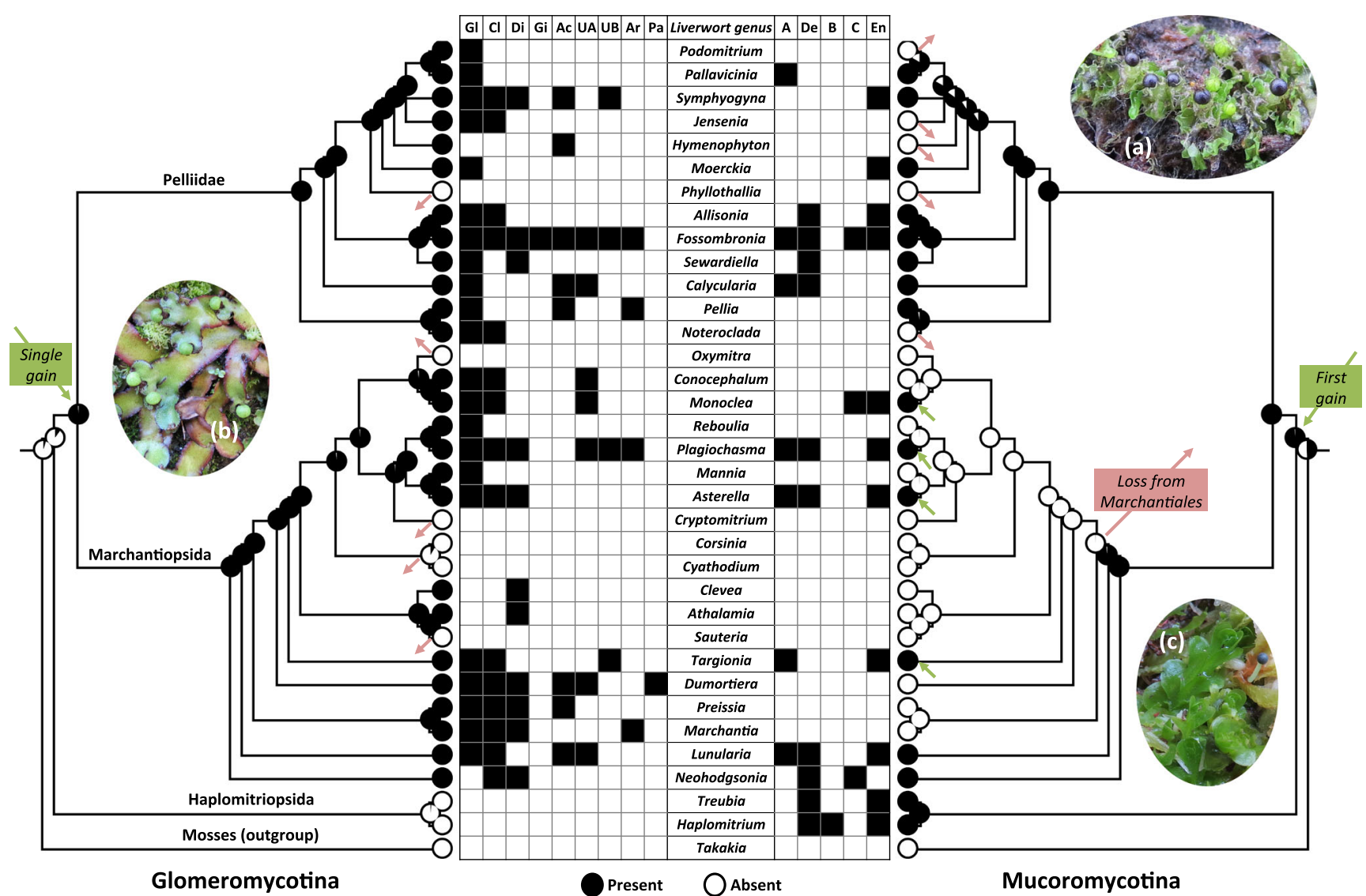

Fig. 2 Ancestral reconstruction of fungal symbiosis in liverworts. The trees are mirror images of a Bayesian inference phylogeny produced using $26 \mathrm{~S}$, the trnK-psbA spacer, and the phylogenies of Forrest et al. (2006) and Flores et al. (2017). Ancestral reconstruction was performed using the Markov 1-parameter model. The grid indicates the presence of different Glomeromycotina and Mucoromycotina families. Family initials represent Gl, Glomeraceae; Cl, Claroideoglomeraceae; Di, Diversisporaceae; Gi, Gigasporaceae; Ac, Acaulosporaceae; UA, Undescribed Archaeosporales A; UB, Undescribed Archaeosporales B;

Ar, Archaeosporaceae; Pa, Paraglomeraceae; A, Putative new Endogonales family A (Fig. 1); De, Densosporaceae; B, Putative new Endogonales family B; C, Putative new Endogonales family C; En, Endogonaceae. The gain and loss events are highlighted by green (gain) and pink (loss) arrows. See also Fig. S3. Examples of the different liverwort groups; (a) Pelliidae - Fossombronia foveolata, (b) Marchantiopsida - Asterella australis, (c) Haplomitriopsida - Treubia pygmaea. Note that the liverwort genus Preissia is now subsumed into Marchantia (Long et al. 2016)

Mucoromycotina also enter into symbiosis with Glomeromycotina. The Mucoromycotina detected in Haplomitriopsida are generalists and of the nine epMT found in these liverworts, only one was Haplomitriopsida-specific. However, the Haplomitriopsida harbor a relatively low diversity of Mucoromycotina compared with the other liverwort groups - nine epMT vs. 15 in Marchantiopsida vs. 22 in Pelliidae. This pattern is maintained when sampling effort is taken into account; the number of epMT detected per colonized sample was 0.2 for Haplomitriopsida, 0.3 for Marchantiopsida, and 0.4 for Pelliidae. Despite also being colonized by Glomeromycotina, Fossombronia (Pelliidae) and Asterella (Marchantiopsida) were colonized by a larger number of epMT than Treubia and Haplomitrium combined. Thus, Haplomitriopsida exhibit specificity to a limited number of Mucoromycotina. It should be noted that in addition to Endogonaceae and Densosporaceae, the phylogenetic trees produced in this study, and by Desirò et al. (2017) when reclassifying the Endogonales, suggest there may be up to three additional families (Fig. 1).

Unlike Haplomitriopsida, Marchantiopsida and Pelliidae liverworts were regularly colonized by both Mucoromycotina and Glomeromycotina and we have increased the number of species confirmed to be colonized by both lineages from two (Field et al. 2016) to 28. This may provide a counterexample to a recent report that dual colonizations by different fungal lineages are rare and unstable (Werner et al. 2018); not only was dual colonization common, but also the ancestral positions of these symbionts (Fig. 2) indicate that these symbioses can be considered evolutionarily stable. In liverworts, co-colonization has been shown to be nutritionally more beneficial than colonization by only one lineage, which may explain why Mucoromycotina symbiosis has been maintained in these plants despite the global dominance of Glomeromycotina (Field et al. 2016, 2019). The 


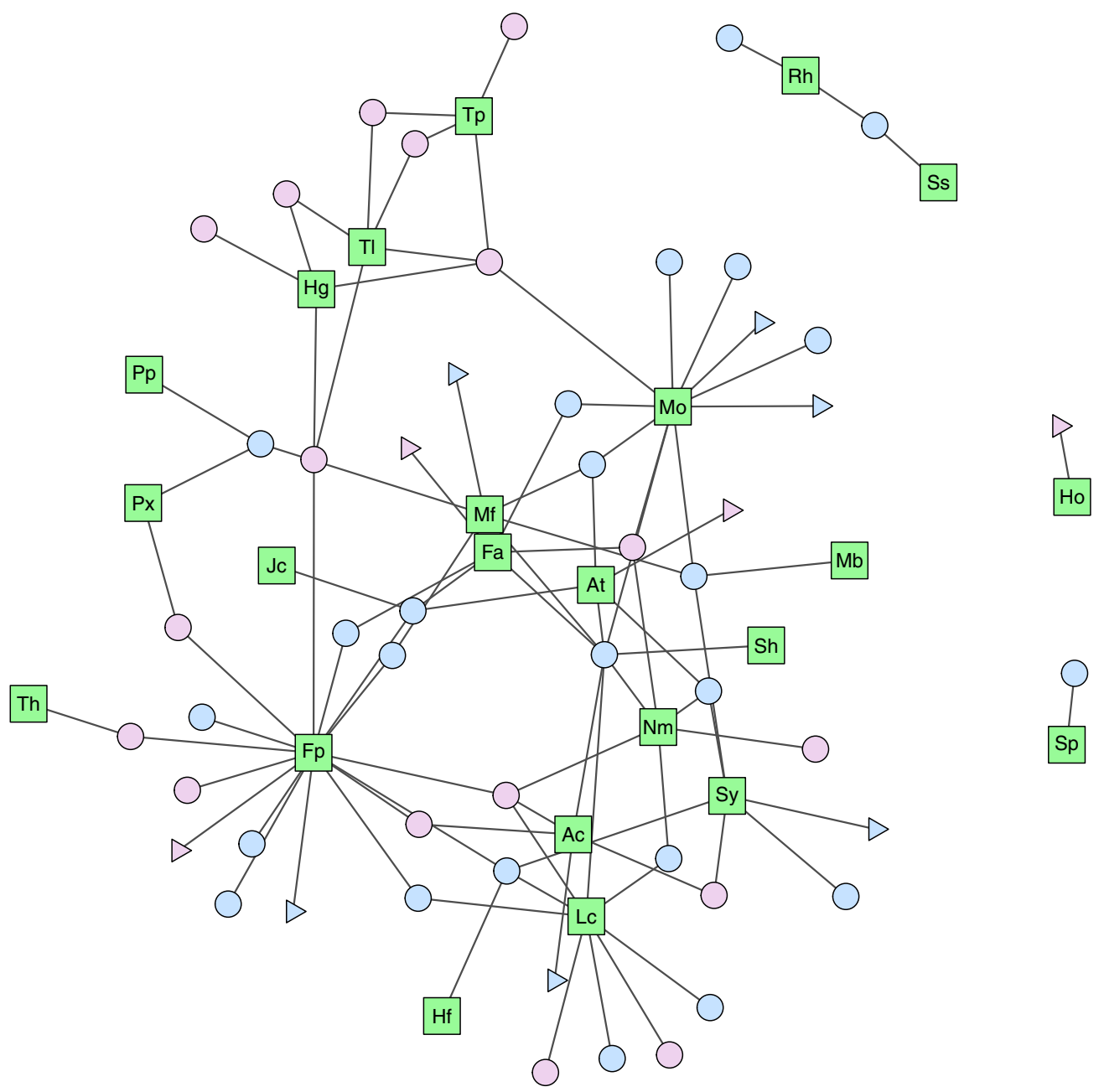

Fig. 3 Network shared between liverworts of the South Island of New Zealand and Mucoromycotina and Glomeromycotina fungi. Green square nodes denote liverworts; blue and pink circles are Glomeromycotina and Mucoromycotina, respectively. Singletons are presented as triangles. Initials represent Ac, Allisonia cockaynei; At, Asterella tenera; $\mathrm{Fa}$, Fossombronia australis; Fp, Fossombronia pusilla; Hg, Haplomitrium gibbsiae; Ho, Haplomitrium ovalifolium; Hf, Hymenophyton flabellatum; Jc, Jensenia connivens; Lc, Lunularia cruciata; Mb,

Glomeromycotina found in co-colonized individuals were disproportionately members of the "older," non-Glomeraceae families. We have previously shown that $36 \%$ of Glomeromycotina colonized liverworts harbor exclusively non-Glomeraceae (Rimington et al. 2018). However, this value increases to $54 \%$ when considering only co-colonized samples. This may hint towards co-colonization being an ancient phenomenon thus supporting the hypothesis that dual symbioses were utilized by early land plants (Field et al. 2015a).

\section{The origins of fungal symbiosis in liverworts}

Ancestral reconstruction supports Mucoromycotina symbiosis evolving before Glomeromycotina symbiosis in liverworts.
Marchantia berteroana; Mf, Marchantia foliacea; Mo, Monoclea forsteri; Nm, Neohodgsonia mirabilis; Px, Pallavicinia xiphoides; Pp, Podomitrium phyllanthus; Rh, Reboulia hemisphaerica; Sh, Symphyogyna hochstetteri; Sy, Symphyogyna hymenophyllum; Sp, Symphyogyna prolifera; Ss, Symphyogyna subsimplex; Th, Targionia hypophylla; Tl, Treubia lacunosa; Tp, Treubia pygmaea. Labels for epMT and epGT are not included but can be seen in Fig. S4

Given current uncertainties surrounding the order of divergence of the three bryophyte lineages (liverworts, hornworts, and mosses) and the monophyly of bryophytes (Puttick et al. 2018; de Sousa et al. 2019), it is not yet possible to confirm or otherwise refute the recent, novel hypothesis, based on multidisciplinary evidence, of Mucoromycotina having formed the ancestral plant-fungus symbiosis (Bidartondo et al. 2011; Field et al. 2015a; Feijen et al. 2018).

The reconstruction has also suggested only one gain of Glomeromycotina in liverworts. This was followed by some losses of symbiosis but never reversion to Glomeromycotina. This pattern of single gain followed by loss without reversion is mirrored by arbuscular mycorrhizal seed plants (Maherali et al. 2016) likely resulting from loss of symbiosis genes (Delaux et al. 2014). In early-diverging liverworts, as in seed 
Table 2 Network analysis results

\begin{tabular}{llll}
\hline & Combined-network & Glomeromycotina-only & Mucoromycotina-only \\
\hline No. liverwort species & 23 & 18 & 14 \\
No. fungal taxa (incl. singletons) & 51 & 30 & 21 \\
Total no. associations & 148 & 69 & 79 \\
Connectance & $8 \%$ & $11 \%$ & $13 \%$ \\
Nestedness & & & \\
NODF$_{\text {full }}$ & 14.68 & 18.36 & 13.44 \\
Nested? & Yes $(p=0.018)$ & No $(p=0.087)$ & No $(p=0.23)$ \\
NODF & 12.50 & 19.53 & 9.65 \\
Nested? & 12.50 & Yes $(p=0.0005)$ & No $(p=0.11)$ \\
NODF & Yes $(p=0.0065)$ & 17.95 & 15.08 \\
Nested? & 15.12 & Yes $(p=0.0012)$ & No $(p=0.27)$ \\
T & Yes $(p<0.00001)$ & $18.09^{\circ}$ & $31.45^{\circ}$ \\
Nested? & $17.8^{\circ}$ & Yes $(p=0.0010)$ & No $(p=0.30)$ \\
BR & Yes $(p=0.0069)$ & 37 & 24 \\
Nested? & 69 & Yes $(p=0.040)$ & No $(p=0.16)$ \\
Modularity & No $(p=0.068)$ & & 6 \\
No. modules & 10 & 0.57 & 0.62 \\
Modularity score & 0.61 & No $(p=0.42)$ & No $(p=0.29)$ \\
Significant? & No $(p=0.06)$ & & \\
\hline
\end{tabular}

The programs ANINHADO and NeD produced the same results for nestedness and significance so only the results of $\mathrm{NeD}$ are presented with the exception of $\mathrm{NODF}_{\text {full }}$, where the $p$ value from ANINHADO is included, as this cannot be calculated by $\mathrm{NeD}$

plants, there must be selection pressure to maintain Glomeromycotina symbiosis. This pressure is not the same for Mucoromycotina symbiosis, which was lost and then gained at least four times (Fig. 2). The Mucoromycotina taxa colonizing liverwort genera that reverted to Mucoromycotina symbiosis are the same as those in plants that have maintained the symbiosis. Thus, these appear to be true reversions and not novel forms of Mucoromycotina symbiosis. Loss and regain of Mucoromycotina endosymbiosis has also occurred in hornworts (Desirò et al. 2013), ferns, and probably lycophytes (Rimington et al. 2015). In contrast, the taxa that form ECM in angiosperms are distinct from those that form endosymbiosis and represent a new form of symbiosis (Fig. S5). In all instances, the liverwort genera that have re-established Mucoromycotina symbiosis have maintained Glomeromycotina symbiosis and there have been no cases of Mucoromycotina being re-established in a non-symbiotic genus. This could stem from these plants likely utilizing the same or similar gene pathways during the establishment of both Mucoromycotina and Glomeromycotina symbiosis. In fact, three symbiosis (sym) genes from Haplomitriopsida liverworts (which exclusively enter into Mucoromycotina symbioses) rescue Glomeromycotina symbiosis in a Medicago truncatula mutant lacking these genes (Wang et al. 2010). To be maintained, sym genes must be functional or they would degrade as in non-symbiotic mosses (Wang et al. 2010). It cannot be ruled out that the sym genes have other, non- symbiotic functions (Bonfante and Selosse 2010), but the work of Wang et al. (2010) strongly supports that at least some of the same genes are used by plants for both Mucoromycotina and Glomeromycotina symbioses. This would explain how Mucoromycotina symbiosis can be reestablished and why, if both types of symbiosis are lost, then neither can be re-established.

The widespread occurrence of Mucoromycotina symbiosis in early-diverging liverworts and nutrient exchange studies (Field et al. 2015b, 2016, 2019) indicate that this relationship can be beneficial to liverworts. It is unknown why Mucoromycotina symbiosis has been lost from some liverworts that have maintained Glomeromycotina symbiosis (Fig. 2). A possible evolutionary scenario for this is related to the higher levels of dual colonization recorded in Pelliidae compared with Marchantiopsida and a single loss of Mucoromycotina symbiosis. Early during the evolution of the Marchantiopsida ca. 196 MYA (Villarreal et al. 2016), there was a complete loss of Mucoromycotina symbiosis (Fig. 2). This was the only major loss of either Mucoromycotina or Glomeromycotina detected (Fig. 2) and resulted in the common ancestor of the largest early-diverging liverwort order (Marchantiales) not entering into symbiosis with Mucoromycotina. As such, for members of this order to form Mucoromycotina symbioses, they first needed to evolve mechanisms to re-establish the relationship. Subsequently, unlike Glomeromycotina, Mucoromycotina is limited to only a 
few Marchantiales genera. The Pelliidae has experienced no major loss of Mucoromycotina during diversification; therefore, the symbiosis is more widespread throughout the group than Marchantiopsida and dual colonization is more common. Additionally, the Pelliidae genera for which Mucoromycotina was not detected were sampled a limited number of times and colonization of Pelliidae liverworts by Mucoromycotina may be as common as it is for Glomeromycotina (Table 1). Thus, the absence of Mucoromycotina symbiosis from genera that enter into Glomeromycotina symbiosis may be solely due to a single loss of Mucoromycotina from the common ancestor of Marchantiales liverworts. The reason for this loss is unknown.

\section{Distinctive symbiotic networks}

To our knowledge, this is the first time network analysis has been performed on Mucoromycotina and is also the first investigation of the symbiotic fungal networks of non-seed plants. When the associations of Mucoromycotina and Glomeromycotina were analyzed together (combined-network), the network appeared to have low connectance and significant nestedness. However, when the fungi were analyzed separately, the network analysis results appeared different; significant nestedness was recorded for the Glomeromycotina-only network but no nestedness was found in the Mucoromycotina-only network (Table 2). Mutualistic networks, such as those between plants and their animal pollinators and seed dispersers, are commonly nested (Bascompte et al. 2003; Bascompte and Jordano 2007; Thébault and Fontaine 2010); however, recent attempts at analyzing plant-fungal mutualistic networks have revealed considerable variation in their structure, depending on mycorrhizal type (Roy-Bolduc et al. 2016). While analyses of plantAM networks showed significant nestedness (Chagnon et al. 2012; Montesinos-Navarro et al. 2012; Chen et al. 2017), those of ectomycorrhizal networks revealed these to be unnested (Bahram et al. 2014; Roy-Bolduc et al. 2016). Thus, the absence of nestedness in our Mucoromycotina-only network is interesting, as it suggests that the networks shared by Mucoromycotina and liverworts may be more like ectomycorrhizal networks than arbuscular mycorrhizal networks. This notion coincides with latest isotope tracer experiments showing that Mucoromycotina, unlike Glomeromycotina, are able to transfer significant amounts of organic nitrogen to liverwort hosts, on a par with ECM (Field et al. 2019). Furthermore, unlike Glomeromycotina, some Mucoromycotina fungi can form ECM (Walker 1985; Yamamoto et al. 2017). The absence of nestedness indicates that Mucoromycotina symbioses in liverworts have greater plasticity than Glomeromycotina symbioses likely resulting from the different lifestyles of the fungal symbionts; Glomeromycotina are limited to obligate endo-biotrophy while Mucoromycotina have options including endobiotrophy, ecto-biotrophy, and saprotrophy. These Mucoromycotina nutritional options, combined with the variable symbiotic status of liverworts, perhaps show that both the symbiont and host may "choose" whether to engage in symbiosis depending upon their growth conditions. For example, unlike all Glomeromycotina, some Mucoromycotina species are not restricted to an obligate lifestyle, so they have a readily available carbon source in the soil and there may be no benefit from entering into symbiosis with a plant, and vice versa for plants with ready access to mineral nutrients (as in vascular plants that are facultatively mycorrhizal with Glomeromycotina). This flexibility is not only a character of these organisms today but was also likely important during plant terrestrialization (Field et al. 2015a). It is worth pointing out that initial ancestral reconstruction analyses - albeit severely constrained by the availability and quality of datasuggest that Mucoromycotina fungi, unlike Glomeromycotina, switched trophic lifestyles (see Supplementary material, Fig. S5).

It is unlikely that the Mucoromycotina-only network contains too few species to produce significant nestedness, but to increase the number of species, we ran analyses again including New Zealand South Island data on Mucoromycotina and Glomeromycotina in hornworts from Desirò et al. (2013) (Table S2b). Species delimitation methods showed the Mucoromycotina that colonize hornworts are the same as those that colonize liverworts, so it is appropriate to combine these data. This increased the number of plant and fungal species to 49, but the network remained un-nested (Table S2b). As this number of species is higher than the number that supports significant nestedness in the Glomeromycotina-only network, we deduce that the lack of significance recorded is not the result of lower species number. Incidentally, when these data from hornworts were included in the Glomeromycotina-only and combined-networks, the support for nestedness was either the same or increased, further validating the use of these additional data. The connectance observed in the Glomeromycotina-only network was the same as previously recorded for a network between $\mathrm{AM}$ and flowering plants, suggesting that the liverwort network is functioning in a similar manner (Montesinos-Navarro et al. 2012). Ultimately, our analyses suggest that Mucoromycotina symbiosis with bryophytes should not be viewed as a type of arbuscular mycorrhiza formed by a fungal lineage different from Glomeromycotina, but as a distinctive symbiosis.

Fossombronia pusilla appeared as the most important member of all three networks and was the only connector hub in all three. Without $F$. pusilla, the network structures would have been different. This important network position stems from the ability of Fossombronia species to enter into the most diverse interactions with both Glomeromycotina and Mucoromycotina (Figs. 1 and 2). Furthermore, they were the most frequently co-colonized plants throughout this study. 
These results suggest that Fossombronia may be pivotal for conserving both Mucoromycotina and Glomeromycotina diversity. A likely explanation may be that the genus contains species ranging from fugitive ephemerals to those forming perennial colonies (Blockeel et al. 2014).

This pioneering study of the Mucoromycotina symbiosis has numerous caveats that are likely to affect all analyses. Here, we only mention some of the main caveats to qualify interpretation of our inferences and to inform subsequent studies. With increased sample sizes, variation in intraspecific colonization needs investigation because it may reflect detection problems and/or biological characteristics. For instance, plantMucoromycotina interactions may be facultative, except in Haplomitriopsida where their pervasiveness indicates those may be obligate. Small sample sizes, sampling unevenness, and small network dimensions in particular may affect network analysis differently, e.g., regarding nestedness and connectance and these should all be addressed. There is a need for improved calibrations for species delineation analysis and intraspecific DNA sequence variation thresholds for fungi, and testing for the presence of cryptic plant species (e.g., for network hub species), as well as replicating network analysis outside New Zealand. The symbiotic status of all early nodes in plant evolution needs assessment to better infer the status of the most recent common ancestor of plants, and the phylogeny and trophic status of most Endogonales also merit analysis. More complex evolutionary models may potentially quantify variation in loss and gain rates across phylogeny and correlated evolution analysis may inform whether there has been continued co-occurrence of Endogonales and Glomeromycotina symbionts.

\section{Conclusions}

We have shown that Mucoromycotina enter into evolutionarily and geographically widespread symbioses, like Glomeromycotina. However, two important differences emerged. Mucoromycotina symbioses are ancestral and may have evolved multiple times in liverworts, as in hornworts, unlike Glomeromycotina symbioses. Glomeromycotina networks are significantly nested, unlike Mucoromycotina ones. The genetic underpinnings and ecological implications of our findings now merit investigation.

Acknowledgments We thank Sir David Read for collecting samples from Indonesia and Gijsbert Werner for comments. Samples collected from Ascension Island were analyzed by Tatiana Solovieva with support from the Society of Biology and the Imperial College Undergraduate Research Opportunities Programme.

Author contributions Conceptualization, W.R.R., S.P., M.I.B.; methodology, W.R.R. and M.I.B.; resources, S.P. and J.G.D.; investigation, W.R.R; writing, original draft, W.R.R., S.P., J.G.D., K.J.F., and M.I.B.
Funding information WRR was supported by the NERC Doctoral Training Programme (Science and Solutions for a Changing Planet); MIB, SP, and KJF were supported by NERC standard grants NE/ N009665/1 and NE/N00941X/1; worldwide collection of liverworts was facilitated by the Leverhulme Trust who awarded an Emeritus Fellowship to JGD and Early Career Fellowship to SP, and by a Royal Society research grant to KJF (RG150276). KJF is supported by a BBSRC Translational Research Fellowship (BB/M026825/1).

Data availability The DNA sequences produced have been uploaded to GenBank and have the following accession numbers: MH174461MH174649.

\section{Compliance with ethical standards}

Conflict of interest The authors declare that they have no conflict of interest.

Open Access This article is distributed under the terms of the Creative Commons Attribution 4.0 International License (http:// creativecommons.org/licenses/by/4.0/), which permits unrestricted use, distribution, and reproduction in any medium, provided you give appropriate credit to the original author(s) and the source, provide a link to the Creative Commons license, and indicate if changes were made.

\section{References}

Almeida-Neto M, Guimarães P, Guimarães PR, Loyola R, Ulrich W (2008) A consistent metric for nestedness analysis in ecological systems: reconciling concept and measurement. Oikos 117:12271239

Atmar W, Patterson BD (1993) The measure of order and disorder in the distribution of species in fragmented habitat. Oecologia 96:373-382

Bahram M, Harend H, Tedersoo L (2014) Network perspectives of ectomycorrhizal associations. Fungal Ecol 7:70-77

Bascompte J, Jordano P (2007) Plant-animal mutualistic networks: the architecture of biodiversity. Annu Rev Ecol Evol Syst 38:567-593

Bascompte J, Jordano P (2014) Mutualistic networks. Princeton University Press, Princeton

Bascompte J, Jordano P, Melian CJ, Olesen JM (2003) The nested assembly of plant-animal mutualistic networks. Proc Natl Acad Sci U S A 100:9383-9387

Bidartondo MI, Read DJ, Trappe JM, Merckx V, Ligrone R, Duckett JG (2011) The dawn of symbiosis between plants and fungi. Biol Lett 7: 574-577

Blockeel TL, Bosanquet SDS, Hill MO, Preston CD (2014) Atlas of British and Irish bryophytes. Pisces Publications, Newbury

Bonfante P, Selosse MA (2010) A glimpse into the past of land plants and of their mycorrhizal affairs: from fossils to evo-devo. New Phytol 186:267-270

Bouckaert R, Drummond A (2017) bModelTest: Bayesian phylogenetic site model averaging and model comparison. BMC Evol Biol 17:42

Bouckaert R, Heled J, Kuhnert D, Vaughan T, Wu CH, Xie D, Suchard MA, Rambaut A, Drummond AJ (2014) BEAST 2: a software platform for Bayesian evolutionary analysis. PLoS Comput Biol 10: e1003537

Brualdi RA, Sanderson JG (1999) Nested species subsets, gaps, and discrepancy. Oecologia 119:256-264

Brundrett MC, Tedersoo L (2018) Evolutionary history of mycorrhizal symbioses and global host plant diversity. New Phytol 220:11081115 
Chagnon PL, Bradley RL, Klironomos JN (2012) Using ecological network theory to evaluate the causes and consequences of arbuscular mycorrhizal community structure. New Phytol 194:307-312

Chen L, Zheng Y, Gao C, Mi XC, Ma KP, Wubet T, Guo LD (2017) Phylogenetic relatedness explains highly interconnected and nested symbiotic networks of woody plants and arbuscular mycorrhizal fungi in a Chinese subtropical forest. Mol Ecol 26:2563-2575

de Sousa F, Foster PG, Donoghue PCJ, Schneider H, Cox CJ (2019) Nuclear protein phylogenies support the monophyly of the three bryophyte groups (Bryophyta Schimp.) New Phytol 222:565-575

Delaux PM, Varala K, Edger PP, Coruzzi GM, Pires JC, Ane JM (2014) Comparative phylogenomics uncovers the impact of symbiotic associations on host genome evolution. PLoS Genet 10:e1004487

Desirò A, Duckett JG, Pressel S, Villarreal JC, Bidartondo MI (2013) Fungal symbioses in hornworts: a chequered history. Proc R Soc Lond B Biol Sci 280:20130207

Desirò A, Rimington WR, Jacob A, Pol NV, Smith ME, Trappe JM, Bidartondo MI, Bonito G (2017) Multigene phylogeny of Endogonales, an early diverging lineage of fungi associated with plants. IMA Fungus 8:245-257

Drummond AJ, Rambaut A (2007) BEAST: Bayesian evolutionary analysis by sampling trees. BMC Evol Biol 7:214

Edgar RC (2004) MUSCLE: a multiple sequence alignment method with reduced time and space complexity. BMC Bioinformatics 5:1-19

Edgar RC (2016) UNOISE2: improved error-correction for Illumina 16S and ITS amplicon reads. bioRxiv. https://doi.org/10.1101/081257

Edgar RC, Haas BJ, Clemente JC, Quince C, Knight R (2011) UCHIME improves sensitivity and speed of chimera detection. Bioinformatics 27:2194-2200

Feijen F, Vos R, Nuytinck J, Merckx V (2018) Evolutionary dynamics of mycorrhizal symbiosis in land plant diversification. Sci Rep 8:10698

Field KJ, Pressel S, Duckett JG, Rimington WR, Bidartondo MI (2015a) Symbiotic options for the conquest of land. Trends Ecol Evol 30: $477-486$

Field KJ, Rimington WR, Bidartondo MI, Allinson KE, Beerling DJ, Cameron DD, Duckett JG, Leake JR, Pressel S (2015b) First evidence of mutualism between ancient plant lineages (Haplomitriopsida liverworts) and Mucoromycotina fungi and its response to simulated Palaeozoic changes in atmospheric $\mathrm{CO}_{2}$. New Phytol 205:743-756

Field KJ, Rimington WR, Bidartondo MI, Allinson KE, Beerling DJ, Cameron DD, Duckett JG, Leake JR, Pressel S (2016) Functional analysis of liverworts in dual symbiosis with Glomeromycota and Mucoromycotina fungi under a simulated Palaeozoic $\mathrm{CO}_{2}$ decline. ISME J 10:1514-1526

Field KJ, Bidartondo MI, Rimington WR, Hoysted GA, Beerling DJ, Cameron DD, Duckett JG, Leake JR, Pressel S (2019) Functional complementarity of ancient plant-fungal mutualisms: contrasting nitrogen, phosphorus and carbon exchanges between Mucoromycotina and Glomeromycotina fungal symbionts of liverworts. New Phytol 223:908-921. https://doi.org/10.1111/nph.15819

Flores JR, Catalano SA, Muñoz J, Suárez GM (2017) Combined phylogenetic analysis of the subclass Marchantiidae (Marchantiophyta): towards a robustly diagnosed classification. Cladistics 34:517-541

Forrest LL, Davis EC, Long DG, Crandall-Stotler BJ, Clark A, Hollingsworth ML (2006) Unraveling the evolutionary history of the liverworts (Marchantiophyta): multiple taxa, genomes and analyses. Bryologist 109:303-334

Fujisawa T, Barraclough TG (2013) Delimiting species using single-locus data and the Generalized Mixed Yule Coalescent Approach: a revised method and evaluation on simulated data sets. Syst Biol 62: $707-724$

Gardes M, Bruns TD (1993) ITS primers with enhanced specificity for Basidiomycetes - application to the identification of mycorrhizae and rusts. Mol Ecol 2:113-118
Glez-Pena D, Gomez-Blanco D, Reboiro-Jato M, Fdez-Riverola F, Posada D (2010) ALTER: program-oriented conversion of DNA and protein alignments. Nucleic Acids Res 38:W14-W18

Guimarães PR, Guimarães P (2006) Improving the analyses of nestedness for large sets of matrices. Environ Model Softw 21:1512-1513

Guimera R, Amaral LAN (2005) Functional cartography of complex metabolic networks. Nature 433:895-900

Guimera R, Sales-Pardo M, Amaral LA (2004) Modularity from fluctuations in random graphs and complex networks. Phys Rev E 70: 025101

Hibbett DS, Binder M, Bischoff JF, Blackwell M, Cannon PF, Eriksson OE, Huhndorf S, James T, Kirk PM, Lucking R et al (2007) A higher-level phylogenetic classification of the Fungi. Mycol Res 111:509-547

Hoysted GA, Kowal J, Jacob A, Rimington WR, Duckett JG, Pressel S, Orchard S, Ryan MH, Field KJ, Bidartondo MI (2018) A mycorrhizal revolution. Curr Opin Plant Biol 44:1-6

Huelsenbeck JP, Ronquist F (2001) MRBAYES: Bayesian inference of phylogenetic trees. Bioinformatics 17:754-755

Kapli P, Lutteropp S, Zhang J, Kobert K, Pavlidis P, Stamatakis A, Flouri $\mathrm{T}$ (2017) Multi-rate Poisson tree processes for single-locus species delimitation under maximum likelihood and Markov chain Monte Carlo. Bioinformatics 33:1630-1638

Kearse M, Moir R, Wilson A, Stones-Havas S, Cheung M, Sturrock S, Buxton S, Cooper A, Markowitz S, Duran C, Thierer T, Ashton B, Meintjes P, Drummond A (2012) Geneious basic: an integrated and extendable desktop software platform for the organization and analysis of sequence data. Bioinformatics 28:1647-1649

Kumar S, Stecher G, Tamura K (2016) MEGA7: molecular evolutionary genetics analysis version 7.0 for bigger datasets. Mol Biol Evol 33: 1870-1874

Ligrone R, Carafa A, Lumini E, Bianciotto V, Bonfante P, Duckett JG (2007) Glomeromycotean associations in liverworts: a molecular, cellular, and taxonomic analysis. Am J Bot 94:1756-1777

Long DG, Forrest LF, Villarreal JC, Crandall-Stotler BJ (2016) Taxonomic changes in Marchantiaceae, Corsiniaceae and Clevaceae (Marchantiidae, Marchantiophyta). Phytotaxa 252:77-80

Maddison WP, Maddison DR (2017) Mesquite: a modular system for evolutionary analysis. Version $3.31 \mathrm{http}: / /$ mesquiteproject.org

Maherali H, Oberle B, Stevens PF, Cornwell WK, McGlinn DJ (2016) Mutualism persistence and abandonment during the evolution of the mycorrhizal symbiosis. Am Nat 188:E113-E125

Miller MA, Pfeiffer W, Schwartz T (2010) Creating the CIPRES Science Gateway for inference of large phylogenetic trees. Proceedings of the Gateway Computing Environments Workshop (GCE):1-8

Montesinos-Navarro A, Segarra-Moragues JG, Valiente-Banuet A, Verdu M (2012) The network structure of plant-arbuscular mycorrhizal fungi. New Phytol 194:536-547

Olesen JM, Bascompte J, Dupont YL, Jordano P (2007) The modularity of pollination networks. Proc Natl Acad Sci U S A 104:1989119896

Öpik M, Davison J, Moora M, Zobel M (2014) DNA-based detection and identification of Glomeromycota: the virtual taxonomy of environmental sequences. Botany 92:135-147

Orchard S, Standish RJ, Dickie IA, Renton M, Walker C, Moot D, Ryan MH (2017a) Fine root endophytes under scrutiny: a review of the literature on arbuscule-producing fungi recently suggested to belong to the Mucoromycotina. Mycorrhiza 27:619-638

Orchard S, Hilton S, Bending GD, Dickie IA, Standish RJ, Gleeson DB, Jeffery RP, Powell JR, Walker C, Bass D, Monk J, Simonin A, Ryan MH (2017b) Fine endophytes (Glomus tenue) are related to Mucoromycotina, not Glomeromycota. New Phytol 213:481-486

Pirozynski KA, Malloch DW (1975) The origin of land plants: a matter of mycotrophism. Biosystems 6:153-164 
Pressel S, Bidartondo MI, Ligrone R, Duckett JG (2010) Fungal symbioses in bryophytes: new insights in the twenty first century. Phytotaxa 9:238-253

Puttick MN, Morris JL, Williams TA, Cox CJ, Edwards D, Kenrick P, Pressel S, Wellman CH, Schneider H, Pisani D, Donoghue PCJ (2018) The interrelationships of land plants and the nature of the ancestral embryophyte. Curr Biol 28:733-745

Rambaut A (2012) FigTree. Version 1.4 http://tree.bio.ed.ac.uk/software/ figtree

Rambaut A, Suchard MA, Xie D, Drummond AJ (2014) Tracer. Version $1.6 \mathrm{http}: / /$ beast.bio.ed.ac.uk/Tracer

Rensing SA (2018) Plant evolution: phylogenetic relationships between the earliest land plants. Curr Biol 28:R210-R213

Renzaglia KS, Villarreal JC, Garbary DJ (2018) Morphology supports the setaphyte hypothesis: mosses plus liverworts forma natural group. Bry Divers Evol 40:011-017

Rimington WR, Pressel S, Duckett JG, Bidartondo MI (2015) Fungal associations of basal vascular plants: reopening a closed book? New Phytol 205:1394-1398

Rimington WR, Pressel S, Duckett JG, Field KJ, Read DJ, Bidartondo MI (2018) Ancient plants with ancient fungi: liverworts associate with early-diverging arbuscular mycorrhizal fungi. Proc R Soc Lond B Biol Sci 285:1888

Roy-Bolduc A, Laliberté E, Hijri M (2016) High richness of ectomycorrhizal fungi and low host specificity in a coastal sand dune ecosystem revealed by network analysis. Ecol Evol 6:349-362

RStudio Team (2015) RStudio: integrated development for R. version 0.99. http://www.rstudio.com/

Schüßler A, Schwarzott D, Walker C (2001) A new fungal phylum, the Glomeromycota: phylogeny and evolution. Mycol Res 105:14131421

Smit E, Leeflang P, Glandorf B, van Elsas JD, Wernars K (1999) Analysis of fungal diversity in the wheat rhizosphere by sequencing of cloned PCR-amplified genes encoding 18S rRNA and temperature gradient gel electrophoresis. Appl Environ Microbiol 65:2614-2621

Smith SE, Read DJ (2008) Mycorrhizal symbiosis. Third edition. Academic Press, Cambridge

Soderstrom L, Hagborg A, von Konrat M, Bartholomew-Began S, Bell D, Briscoe L, Brown E, Cargill DC, Costa DP, Crandall-Stotler BJ et al (2016) World checklist of hornworts and liverworts. Phytokeys 59:1-828

Southworth D, He XH, Swenson W, Bledsoe CS, Horwath WR (2005) Application of network theory to potential mycorrhizal networks. Mycorrhiza 15:589-595

Spatafora JW, Chang Y, Benny GL, Lazarus K, Smith ME, Berbee ML, Bonito G, Corradi N, Grigoriev I, Gryganskyi A, James TY, O'Donnell K, Roberson RW, Taylor TN, Uehling J, Vilgalys R, White MM, Stajich JE (2016) A phylum-level phylogenetic classification of zygomycete fungi based on genome-scale data. Mycologia 108:1028-1046

Stamatakis A (2014) RAxML version 8: a tool for phylogenetic analysis and post-analysis of large phylogenies. Bioinformatics 30:13121313
Stotler RE, Crandall-Stotler B (2017) A synopsis of the liverwort flora of North America north of Mexico. Ann Mo Bot Gard 102:574-709

Strona G, Galli P, Seveson D, Montano S, Fattorini S (2014) Nestedness for Dummies (NeD): a user-friendly web interface for exploratory nestedness analysis. J Stat Softw 59

Taylor TN, Remy W, Hass H, Kerp H (1995) Fossil arbuscular mycorrhizae from the Early Devonian. Mycologia 87:560-573

Tedersoo L, Sánchez-Ramírez S, Kõljalg U, Bahram M, Döring M, Schigel D, May T, ryberg M, Abarenkov K (2018) High-level classification of the Fungi and a tool for evolutionary ecological analyses. Fungal Divers 90:135-159

Thébault E, Fontaine C (2010) Stability of ecological communities and the architecture of mutualistic and trophic networks. Science 329: 853-856

Ulrich W, Almeida-Neto M, Gotelli NJ (2009) A consumer's guide to nestedness analysis. Oikos 118:3-17

van der Heijden MGA, Martin FM, Selosse M-A, Sanders IR (2015) Mycorrhizal ecology and evolution: the past, the present, and the future. New Phytol 205:1406-1423

Villarreal JC, Crandall-Stotler BJ, Hart ML, Long DG, Forrest LL (2016) Divergence times and the evolution of morphological complexity in an early land plant lineage (Marchantiopsida) with a slow molecular rate. New Phytol 209:1734-1746

Walker C (1985) Endogone lactiflua forming ectomycorrhizas with Pinus contorta. Trans Br Mycol Soc 84:353-355

Walker C, Gollotte A, Redecker D (2018) A new genus, Planticonsortium (Mucoromycotina), and new combination (P. tenue), for the fine root endophyte, Glomus tenue (basionym Rhizophagus tenuis). Mycorrhiza 28:213-219

Wang B, Yeun LH, Xue J-Y, Liu Y, Ane J-M, Qiu Y-L (2010) Presence of three mycorrhizal genes in the common ancestor of land plants suggests a key role of mycorrhizas in the colonization of land by plants. New Phytol 186:514-525

Werner GDA, Cornelissen JHC, Cornwell WK, Soudzilovskaia NA, Kattge J, West SA, Kiers ET (2018) Symbiont switching and alternative resource acquisition strategies drive mutualism breakdown. Proc Natl Acad Sci U S A 115:5229-5234

White TJ, Bruns T, Lee S, Taylor JW (1990) Amplification and direct sequencing of fungal ribosomal RNA genes for phylogenetics. In: Innis MA, Gelfand DH, Sninsky JJ, White TJ (eds) PCR protocols: a guide to methods and applications. Academic Press, Orlando, pp 315-322

Yamamoto K, Endo N, Degawa Y, Fukuda M, Yamada A (2017) First detection of Endogone ectomycorrhizas in natural oak forests. Mycorrhiza 27:295-301

Zhang J, Kapli P, Pavlidis P, Stamatakis A (2013) A general species delimitation method with applications to phylogenetic placements. Bioinformatics 29:2869-2876

Publisher's note Springer Nature remains neutral with regard to jurisdictional claims in published maps and institutional affiliations. 